\title{
Spontaneous Acute Subdural Hemorrhage with Rupture of Intracranial Aneurysm: A Series of Two Cases and Review of Literature
}

\author{
Amol Raheja ${ }^{1}$ Sumit Bansal ${ }^{2}$ Sachin A. Borkar ${ }^{1}$ Ashish Suri ${ }^{1}$ Ashok K. Mahapatra ${ }^{2}$ \\ ${ }^{1}$ Department of Neurosurgery, All India Institute of Medical Sciences, \\ New Delhi, India \\ 2 Department of Neurosurgery, All India Institute of Medical Sciences, \\ Bhubaneswar, Orissa, India \\ Address for correspondence Ashish Suri, MCh, Department of
Neurosurgery, Room No. 712, Neurosciences Center, All India
Institute of Medical Sciences, New Delhi 110029, India
(e-mail: surineuro@gmail.com; surineuro@hotmail.com).
}

Indian J Neurosurg 2016;5:16-21.
We present a rare series of two cases of spontaneous intracranial aneurysmal hemorrhage associated with acute subdural hematoma. One patient was having ruptured anterior communicating artery aneurysm and acute subdural hematoma with no evidence of subarachnoid hemorrhage and the other patient presented with subarachnoid hemorrhage with bleeding in subdural, intraparenchymal, and intraventricular location with rupture of posterior communicating artery aneurysm. In this report, we are describing the pathophysiology and radiological features of subdural hematoma with aneurysm bleed. hematoma

\section{Introduction}

Subarachnoid hemorrhage (SAH) almost invariably develops following the rupture of a cerebral aneurysm, and is often complicated by intracerebral hematoma (ICH) and intraventricular hematoma. Subdural hematoma (SDH) can occur rarely. Reported incidence of SDH associated with aneurysm bleed varies from 0.5 to $7.9 \% .^{1,2}$ In most cases SDH is associated with SAH and/or ICH. Intracranial aneurysmal rupture may cause acute $\mathrm{SDH}$ with or without radiologic evidences of $\mathrm{SAH}^{3,4}$ In this report, we are describing the pathophysiology and radiologic features of SDH with aneurysm bleed.

\section{Case Reports}

\section{First Case}

An 18-year-old man presented with complaints of generalized seizure associated with severe headache and weakness of all four limbs (power 4/5). There was no history suggestive of trauma. Noncontrast computed tomography (NCCT) of the head on admission did not reveal any abnormality (-Fig. 1A). Magnetic resonance imaging (MRI) of the brain on admission was suggestive of features of suspected posterior circulation stroke. The patient was evaluated and admitted under neurology. Extensive vasculitis and coagulation workup failed to identify the etiology. Repeat NCCT of the head done after 1 week revealed evidence of bilateral thalamic ischemic changes (-Fig. 1B).

The patient had recurrence of seizure after 1 week with radiologic evidence of new onset isolated thin right frontoparietal convexity acute SDH reaching till falx cerebri and tentorium cerebelli with no evidence of subarachnoid or intracerebral hemorrhage (-Fig. 1C). He also had intermittent fever spikes and vomiting subsequently. Fever workup for malarial antigen and enteric fever were negative and blood culture was sterile. Urine culture was positive for pseudomonas species, sensitive to piperacillin and tazobactam. The patient was managed conservatively with antiepileptics, culture-based antibiotics, and antipyretics. Inability to achieve a final diagnosis prompted to obtain a six-vessel intra-arterial digital subtraction angiography
License terms of India
April 10, 2015

accepted

November 18, 2015

published online

February 22, 2016
DOI http://dx.doi.org/

10.1055/s-0036-1572369.

ISSN 2277-954X. 


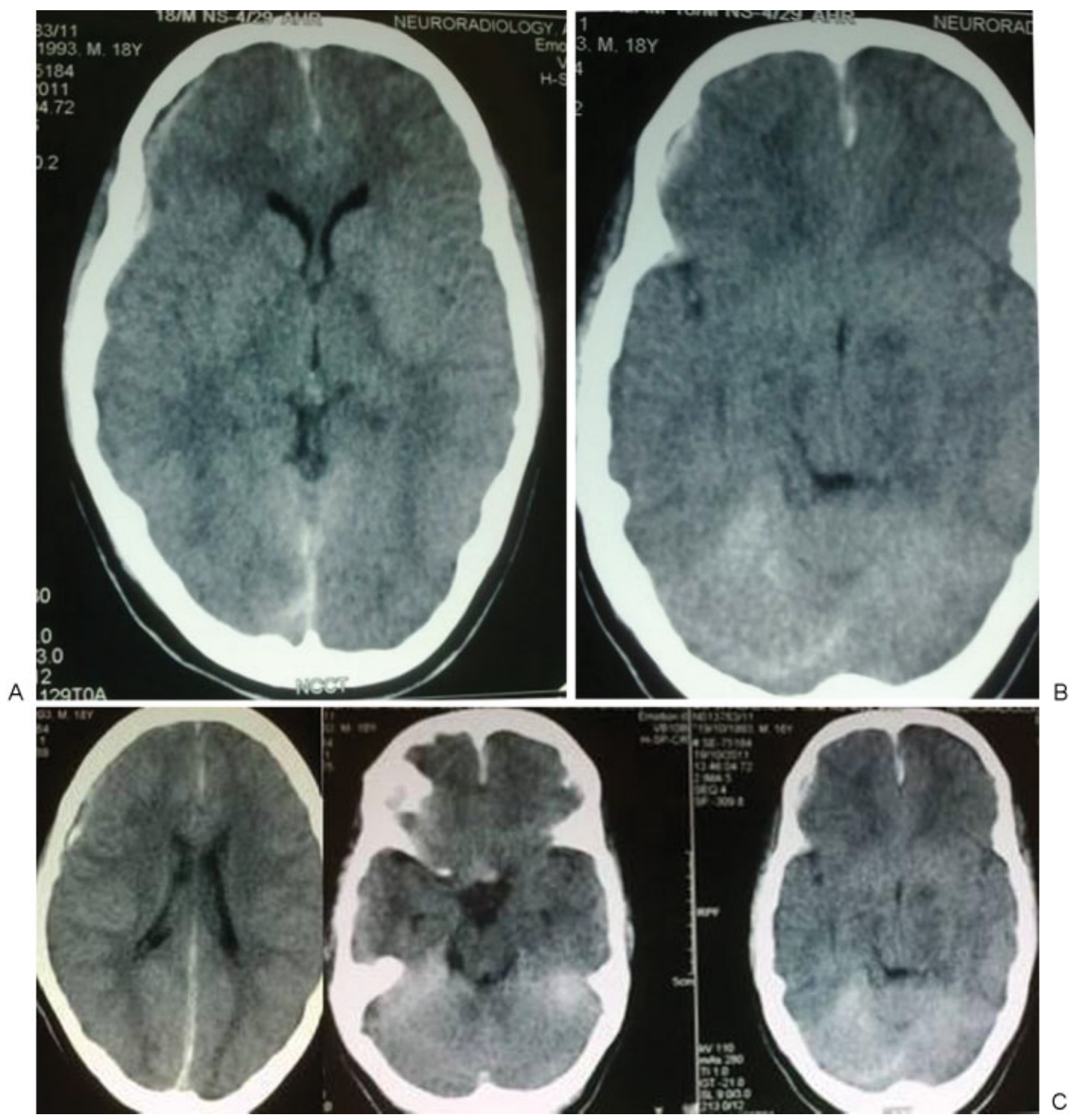

Fig. 1 First case: Normal NCCT of the head on admission (A). NCCT of the head (after 1 week): B/L thalamic ischemic changes (B). NCCT of the head (after 2 weeks): Thin right frontoparietobasal SDH reaching till falx and tentorium (C). Angiography shows anterior communicating artery aneurysm measuring $8.17 \times 4.35 \mathrm{~mm}$ filling from right side pointing anteroinferomedially with neck measuring $2.4 \mathrm{~mm}$ (D). NCCT of the head (postoperative): evidence of right lateral supraorbital craniotomy and clip artifact. No evidence of $\mathrm{EDH} /$ hematoma/ventriculomegaly seen (E).

(IADSA) that subsequently revealed an $8.17-\times 4.35-\mathrm{mm}$ anterior communicating artery aneurysm (A-Comm), filling from the right side and pointing anteroinferomedially with neck measuring $2.4 \mathrm{~mm}$ (-Fig. 1D). There was no evidence of radiological vasospasm.

The patient was transferred under neurosurgery and was operated electively. Right lateral supraorbital craniotomy was performed. Right frontotemporal SDH was evacuated and A-Comm aneurysm was clipped (-Fig. 1E). Organized subdural frontobasal and parietal hematoma was present on the right side. He had an uneventful postoperative period and was discharged after 1 week with no residual sensorymotor deficits. The patient was clinically asymptomatic on follow-up after 6 weeks. Histopathologic examination revealed fibrocollagenous tissue with fresh and old hemorrhage and infiltration by macrophages.

\section{Second Case}

A 49-year-old woman, with no history of head trauma, presented with 1 day episode of left forehead heaviness followed by sudden headache and vomiting. The patient came to our hospital in unconscious state. On neurologic examination, the Glasgow Coma Scale (GCS) was E1V1M2; pupils were bilateral constricted and sluggish reacting to light.

Neuroimaging showed right frontal extra-axial lesion suggestive of acute SDH with right temporal hematoma and intraventricular bleed (-Fig. 2A). A CT angiogram demonstrated right post communicating artery aneurysm (-Fig. 2B).

The patient could not be taken up for operative procedure because of deranged coagulation profile and very poor clinical status. Ideally, endovascular treatment should have been done in view of inability to clip the aneurysm because 


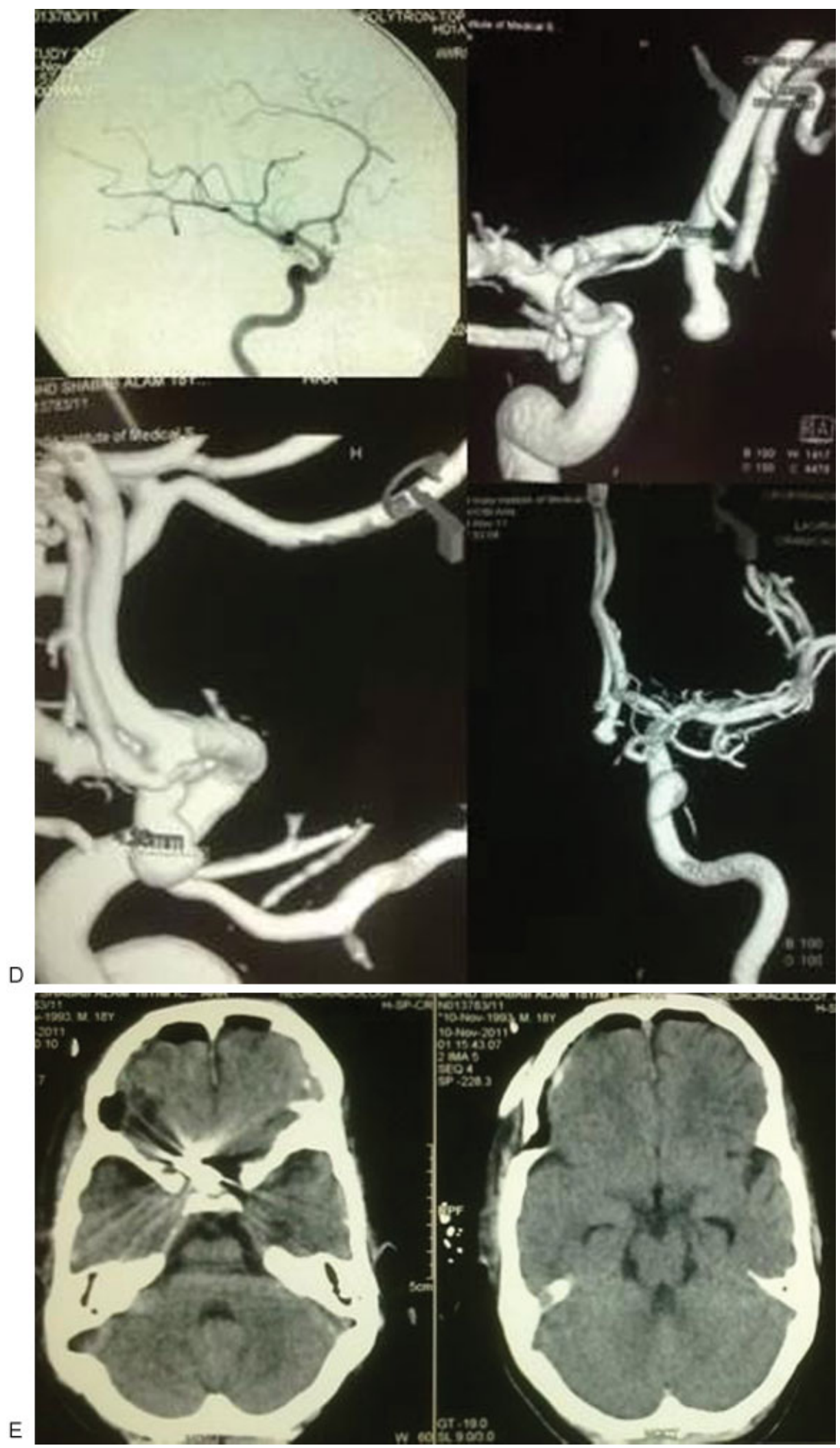

Fig. 1 Continued 

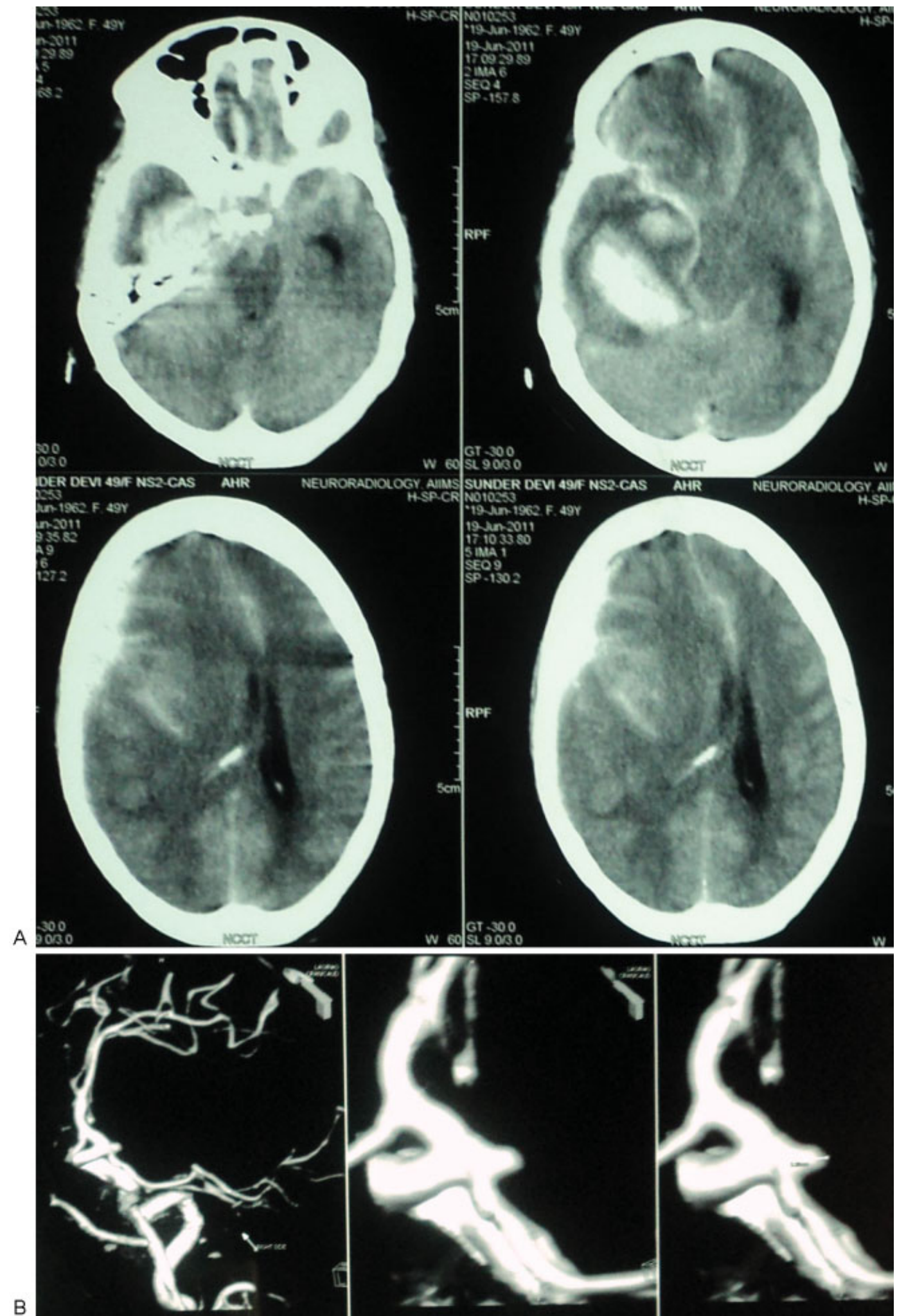

Fig. 2 Second case: Axial CT images show right frontotemporal subdural hematoma with right temporal hematoma with gross subarachnoid hemorrhage with intraventricular extension with marked midline shift toward left (A). Right common carotid angiography shows a large aneurysm of the right posterior communicating artery segment (B). 
Table 1 Differentiation of aneurysmal and traumatic subdural hematoma

\begin{tabular}{|l|l|l|l|}
\hline S no. & Feature & Traumatic SDH & $\begin{array}{l}\text { Spontaneous acute SDH } \\
\text { (aneurysmal) }\end{array}$ \\
\hline 1. & History of trauma & Present & Absent \\
\hline 2. & CT characteristics & $\begin{array}{l}\text { - Mixed, iso- or hypodense } \\
\text { May be bilateral } \\
\text { Lentiform as well as crescentic }\end{array}$ & $\begin{array}{l}\text { - Hyperdense } \\
\text { Unilateral } \\
\text { - Crescentic in convexity or triangular over } \\
\text { the lower Sylvian fissure }\end{array}$ \\
\hline 3. & Associated CT findings & $\begin{array}{l}\text { Pure SDH or associated with hemorrhagic } \\
\text { contusions with further evidence } \\
\text { of scalp/bone injury }\end{array}$ & $\begin{array}{l}\text { Frequent association with SAH, ICH, } \\
\text { or intraventricular hemorrhage }\end{array}$ \\
\hline
\end{tabular}

Abbreviation: $\mathrm{CT}$, computed tomography; $\mathrm{ICH}$, intracerebral hematoma; $\mathrm{SAH}$, subarachnoid hemorrhage; SDH, subdural hematoma.

of deranged coagulation profile, but the patient's relatives did not agree for endovascular treatment of the aneurysm (because of financial restrictions), so he was managed conservatively.

\section{Discussion}

Only very few cases ( $<20$ cases so far) have been documented in literature for isolated SDH presentation of aneurysm rupture. ${ }^{5-11}$

The three mechanisms are postulated for isolated. First, an aneurysm adherent to the arachnoid may bleed directly into the subdural space. The previous minor bleeds may cause localized arachnoid adhesions to aneurysm dome. Second, aneurysm rupture with hemorrhage under significant pressure may lead to pia-arachnoid rupture and subsequent SDH. Third, aneurysms arising from part of carotid lying in subdural space will eventually cause SDH after rupture.,

Barton and Tudor $^{3}$ studied various aneurysm locations contributing to SDH associated with aneurysmal bleed and $\mathrm{SAH} ; 34.4 \%$ of SDH were due to anterior cerebral/A-Comm aneurysm bleeds. Although previous reports by Strang et $\mathrm{al}^{2}$ in 1961 showed $17 \%$ of such cases associated with A-Comm/ anterior cerebral artery aneurysmal bleeds, the present cases exemplify the significance of aneurysmal etiology of nontraumatic SDH.

Weir et al, in $1982,{ }^{12}$ proposed the following CT criteria to differentiate SDH caused by ruptured aneurysms or trauma: (1) SDH of aneurysmal origin is unilateral, hyperdense, and crescentic in convexity or triangular over the lower Sylvian fissure, whereas traumatic SDH is more likely to be mixed, iso- or hypodense, may be bilateral or lentiform as well as crescentic; (2) SDH due to ruptured aneurysms is frequently associated with SAH, intracerebral hemorrhage, or intraventricular hemorrhage, whereas traumatic SDH may be pure SDH or associated with hemorrhagic contusions with further evidence of scalp or bone injury (-Table 1).

The first case was admitted and managed initially in Neurology Department for about 1 week. Then neurosurgery consultation was sought, and then repeat CT of the head and IADSA were planned and then after confirming aneurysm definitive steps taken. A CT angiogram could also be obtained if IADSA was not feasible. In first case, the patient initially did not reveal SDH on CT scan, which later became prominent after second seizure. This suggests possibility of earlier minor bleed causing arachnoid adhesions and later on manifesting as SDH on rebleed. Although the scans did not show all the characteristics of either aneurysmal or traumatic SDH, but a high index of suspicion is necessary for arriving at the right diagnosis and early management. This case report also reinforces the pitfalls associated with CT in diagnosing SDH caused by ruptured aneurysms and potentially emphasizing on the role of angiography in selected cases with high index on clinical suspicion. ${ }^{13,14}$

Multicompartmental hemorrhage may support the theories that high pressure emanating from the ruptured aneurysm or massive hemorrhage causes an aneurysmal $\mathrm{SDH}$. Three-dimensional CT angiography is regarded as the best investigative technique for differentiating aneurysmal $\mathrm{SDH}$ if the patient is in poor clinical condition. ${ }^{15,16}$ The conventional four-vessel angiography is the gold standard for detecting the presence of an aneurysm; however, it is time consuming and not safer than 3D CT angiography, particularly in poor-grade patients. ${ }^{17}$

\section{Conclusion}

Clinicians should be aware of the possibility of aneurysmal SDH to avoid misdiagnosis and mismanagement. Characteristic CT findings and clinical history may be helpful for differential diagnosis. For early detection of aneurysm even in the patient with poor clinical grade, 3D CT angiography is a very useful tool.

\section{References}

1 Bassett RC, Lemmen LJ. Subdural hematoma associated with bleeding intracranial aneurysm. J Neurosurg 1952;9(5):443-450

2 Strang RR, Tovi D, Hugosson R. Subdural hematomas resulting from the rupture of intracranial arterial aneurysms. Acta Chir Scand 1961;121:345-350

3 Barton E, Tudor J. Subdural haematoma in association with intracranial aneurysm. Neuroradiology 1982;23(3):157-160

4 Han YH, Kang SD, Kim JM. Two cases of posterior communicating artery aneurysm complicated by massive subdural hematoma. J Korean Neurosurg Soc 1996;25:1036-1046

5 Araki T, Sampei T, Murata H, Fujiwara H, Taki W. [A case of internal carotid-posterior communicating artery aneurysm 
presenting pure acute subdural hematoma]. No Shinkei Geka 2002;30(8):861-866

6 Ban S, Sato S, Yamamoto T, Ogata M. [Distal anterior cerebral artery aneurysm causing acute subdural hematoma: report of two cases]. No Shinkei Geka 1985;13(8):911-916

7 Katsuno M, Murai Y, Teramoto A. [Acute subdural hematoma without subarachnoid hemorrhage following rupture of a distal anterior cerebral artery aneurysm: a case report]. No To Shinkei 2003;55(5):435-438

8 Kondziolka D, Bernstein M, ter Brugge K, Schutz H. Acute subdural hematoma from ruptured posterior communicating artery aneurysm. Neurosurgery 1988;22(1 Pt 1):151-154

9 O'Leary PM, Sweeny PJ. Ruptured intracerebral aneurysm resulting in a subdural hematoma. Ann Emerg Med 1986;15(8): 944-946

10 Watanabe K, Wakai S, Okuhata S, Nagai M. Ruptured distal anterior cerebral artery aneurysms presenting as acute subdural hematoma-report of three cases. Neurol Med Chir (Tokyo) 1991; 31(8):514-517

11 Fein JM, Rovit RL. Interhemispheric subdural hematoma secondary to hemorrhage from a calloso-marginal artery aneurysm. Neuroradiology 1970;1:183-186
12 Weir B, Myles T, Kahn M, et al. Management of acute subdural hematomas from aneurysmal rupture. Can J Neurol Sci 1984; 11(3):371-376

13 Handel SF, Perpetuo FO, Handel CH. Subdural hematomas due to ruptured cerebral aneurysms: angiographic diagnosis and potential pitfall for CT. AJR Am J Roentgenol 1978;130(3): 507-509

14 Reichenthal E, Savitz MH, Rothman AS, Katz SS, Malis LI. Ruptured intracranial aneurysms as a cause of subdural haematoma. Potential diagnostic pitfalls and the surgical management of the acute patient. Neurochirurgia (Stuttg) 1986;29(6):219-224

15 Kaminogo M, Hayashi H, Ishimaru H, et al. Depicting cerebral veins by three-dimensional $\mathrm{CT}$ angiography before surgical clipping of aneurysms. AJNR Am J Neuroradiol 2002;23(1):85-91

16 Shenoy SN, Kumar MG, Raja A. Intracranial aneurysms causing spontaneous acute subdural hematoma. Neurol India 2003;51(3): 422-424

17 Hsiang JN, Liang EY, Lam JM, Zhu XL, Poon WS. The role of computed tomographic angiography in the diagnosis of intracranial aneurysms and emergent aneurysm clipping. Neurosurgery 1996;38(3):481-487, discussion 487 